\title{
Examining the unmet need in adults with
}

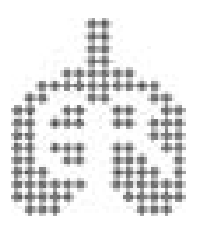

\section{severe asthma}

\author{
M.R. Partridge
}

\begin{abstract}
Asthma currently affects an estimated $\mathbf{3 0 0}$ million people worldwide and the number is expected to rise to 400 million by 2025 . Asthma morbidity remains high and the economic burden is significant. Approximately $20 \%$ of patients have severe persistent asthma. As patients with severe asthma often have a variety of conditions that may coexist with or be mistaken for asthma, careful diagnosis and management are essential, and adhering to a protocol for investigations is helpful.

For patients with severe persistent asthma, the Global Initiative for Asthma 2005 guidelines recommend the use of high-dose inhaled corticosteroids in combination with a long-acting $\beta_{2}-$ agonist, with one or more additional controller medications if required (step 4 therapy). However, recent studies have shown that asthma remains inadequately controlled in many patients with severe asthma, despite treatment in accordance with guidelines.

Patients with severe asthma have the highest healthcare utilisation and mortality, and there is clearly an unmet need for the effective and safe treatment of patients with severe persistent allergic asthma who remain symptomatic despite optimised standard treatment. The latest guidelines suggest that omalizumab may address this unmet need.
\end{abstract}

KEYWORDS: Adults, difficult asthma, severe asthma, unmet needs

$\mathrm{t}$ is estimated that as many as 300 million people of all ages and from all ethnic backgrounds have asthma and the burden of this disease to governments, healthcare systems, families and patients is increasing worldwide. Globally, asthma is one of the most common long-term diseases and considerably higher estimates can be obtained with less conservative criteria for the diagnosis of clinical asthma [1]. The rate of asthma increases as communities adopt Western lifestyles and become urbanised. As the proportion of the world's population that is urban is projected to increase from $45 \%$ in 2004 to $59 \%$ in 2025 , there is likely to be a marked increase in the global prevalence of asthma over the next two decades. Indeed, it is estimated that there may be an additional 100 million people with asthma by 2025 [1]. Asthma mortality is high, with $\sim 239,000$ asthma-related deaths per year [2]. The economic burden of asthma is also considerable, with an estimated total cost of asthma in Europe of $€ 17.7$ billion per year [3].

\section{THE GLOBAL INITIATIVE FOR ASTHMA ASSESSMENT $^{1}$}

The Global Initiative for Asthma (GINA) 2005 guidelines classified asthma severity into four

\footnotetext{
${ }^{1}$ See Post-meeting note on page 88.
}

steps, according to clinical features before treatment (table 1), as well as by the daily medication regimen and the response to treatment (table 2) [4]. Asthma is classified as follows. Step 1: intermittent, with symptoms occurring less than once a week and patients remaining asymptomatic with normal peak expiratory flow (PEF) between attacks. Step 2: mild persistent, with symptoms occurring more than once a week, but with $\leqslant 1$ attack $\cdot$ day $^{-1}$. Step 3: moderate persistent, with daily attacks affecting activity. Step 4: severe persistent, with continuous limited physical activity. Although estimates vary, it is likely that $\sim 25-40 \%$ of patients have intermittent asthma, $25-40 \%$ have mild or moderate persistent asthma, 20\% have severe persistent asthma, and 5-10\% have uncontrolled asthma despite receiving optimised therapy (severe/difficult asthma). Patients who have severe persistent asthma (step 4) experience highly variable continuous symptoms, reduced lung function, frequent nocturnal symptoms, limitations in activities and severe exacerbations despite medication.

\section{DIAGNOSIS CONSIDERATIONS}

Before treating patients with difficult asthma, some other factors should be considered. Misdiagnosis may be as high as $10 \%$, nonadherence with oral steroid therapy has been reported
CORRESPONDENCE

M.R. Partridge Dept of Respiratory Medicine Faculty of Medicine Imperial College London Charing Cross Campus St Dunstans Road London W6 8RP UK Fax: 442088467999 E-mail: m.partridge@imperial.ac.uk

STATEMENT OF INTEREST

M.R. Partridge has received sponsorship to attend international meetings from GlaxoSmithKline AstraZeneca and Novartis Pharma AG and has received lecture fees from AstraZeneca, Novartis Pharma AG, Medeva and Cipla Pharmaceuticals. M.R. Partridge has also received research funding from the Astra Foundation. This issue of the European Respiratory Review contains proceedings of a satellite symposium held at the 16th ERS Annual Congress, 2006, which was sponsored by Novartis Pharma AG. The authors were assisted in the preparation of the text by professiona medical writers at ACUMED $\mathbb{\circledast}$; this support was funded by Novartis Pharma AG. 
at $30 \%$, and in some cases there is a significant major psychiatric component to patients' symptoms and perception of their asthma [5-7]. After excluding these factors, only a half of the cases are truly therapy-resistant asthma or severe asthma (fig. 1) [7]. Therefore, the careful diagnosis and management of patients with severe asthma is necessary, and an approach using defined protocol is often helpful $[8,9]$. In 1998, the European Respiratory Society (ERS) Task Force on difficult/therapy-resistant asthma recommended the use of a protocol for evaluating difficult asthma (table 3) [8]. A similar approach had been recommended earlier, in the USA in 1993 [9]. A protocol is also useful for: addressing and attempting to validate issues raised in the proposed definition; providing data that can be compared between centres; and standardising patient categories for research purposes, including therapeutic trials [8].

Some patients with apparently severe asthma have a variety of comorbidities. As many conditions can induce wheezing associated with airway obstruction, a patient with a presumed diagnosis of asthma, but not apparently responding to asthma medication, should be investigated for other possible alternative or associated diagnoses. Superficially similar conditions may be mistaken for asthma and this may lead to patients being inappropriately prescribed inhaled steroid therapy. For example, although cough and the presence of eosinophils in sputum may be considered to be a variant of asthma, cough alone in the absence of evidence of intermittent bronchial obstruction is unlikely to be caused by asthma. Allergic bronchopulmonary aspergillosis and pulmonary eosinophilic syndromes may be considered unique diseases with some of the clinical features of asthma and are often difficult to treat; these are probably best considered outside the definition of difficult/therapy-resistant asthma. Vocal cord dysfunction (VCD), characterised by adduction of the vocal cords, can masquerade as asthma or may coexist with asthma, which could result in asthma appearing more severe than it really is $[10,11]$. In one study [11], $16.7 \%$ of patients with refractory asthma and referred for tertiary care had coexisting VCD and asthma. Moreover, medical utilisation is higher in patients with VCD compared with age/sex-matched patients with asthma [12]. Other respiratory conditions, such as chronic bronchitis or bronchiectasis, may also coexist with asthma, but the effect of these on asthma severity or control is unknown [8].

A recent survey of members of the British Thoracic Society was conducted to assess the current level of use of protocols for severe asthma. The responses of 21 specialists with a special interest in difficult asthma were compared with those of 152 doctors who were not the lead for asthma or difficult asthma in their institution [13]. Difficult asthma specialists were more likely to always utilise liaison psychiatric opinion $(p=0.002)$, to use prednisolone assays/cortisols to objectively assess compliance $(p=0.028)$ and to routinely perform skin-prick tests for common inhaled allergens and fungi $(p=0.012$ and $p<0.001$, respectively). The most significant difference between specialists and generalists was the increased ease of access to a liaison psychiatrist by referral $(p=0.001)$. The survey concluded that pulmonologists with a declared special interest in difficult asthma may have configured their services and approaches more in line with the recommendations of the ERS Task Force [8]. However, the results also indicated that a protocol-based approach for difficult asthma is not widespread among respiratory physicians who did not have a special interest in asthma.

\section{GINA GUIDELINES AND CONTROL OBJECTIVES ${ }^{1}$}

GINA 2005 guidelines recommend that treatment should be tailored to asthma severity, with defined regimens for each treatment step. If asthma control cannot be achieved or is lost on the current treatment step, treatment should be advanced to the next step (fig. 2) [4]. Patients with intermittent asthma (step 1 ) should receive an inhaled short-acting $\beta_{2}$-agonist (SABA). Patients with mild persistent asthma (step 2) should also receive a low-dose inhaled corticosteroid (ICS). Additionally, an inhaled long-acting $\beta_{2}$-agonist (LABA) may be used in patients with moderate asthma (step 3). For patients with severe persistent asthma, the GINA guidelines recommend the use of high-dose ICS in combination with a LABA, with one or more additional controller medications if required (step 4 therapy). GINA acknowledges that control of asthma may not always be possible. Additional controllers are anti-immunoglobulin (Ig)E, leukotriene modifiers, oral $\beta_{2}$-agonist, oral corticosteroid (OCS) and sustained-release theophylline.

\section{TABLE 1 Global Initiative for Asthma 2005 classification of asthma severity: clinical features before treatment}

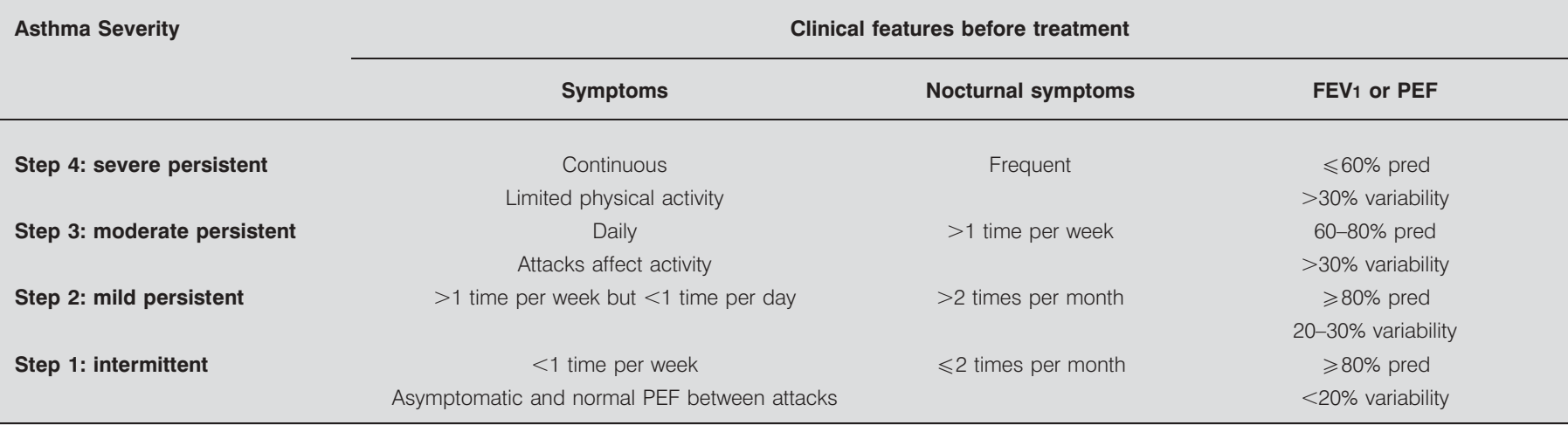

The presence of one feature of severity is sufficient to place a patient in that category. FEV1: forced expiratory volume in one second; \% pred: \% predicted; PEF: peak expiratory flow. Reproduced from [4] with permission from the publisher. 
TABLE 2 Global Initiative for Asthma 2005 classification of asthma severity: clinical features and treatment

\begin{tabular}{|c|c|c|c|c|}
\hline Clinical features & $\begin{array}{c}\text { Step } 1 \\
\text { No controller }\end{array}$ & $\begin{array}{c}\text { Step } 2 \\
<\mathbf{5 0 0 \mu g ~ B D P ~}\end{array}$ & $\begin{array}{c}\text { Step } 3 \\
200-1000 \mu g \text { BDP and } \\
\text { LABA }\end{array}$ & $\begin{array}{c}\text { Step } 4 \\
>1000 \mu \mathrm{g} \mathrm{BDP} \text { and LABA } \\
\text { and/or other }\end{array}$ \\
\hline $\begin{array}{l}\text { Step } 1 \\
\text { Symptoms }<1 \text { time per week } \\
\text { Nocturnal symptoms } \leqslant 2 \text { times per month } \\
\text { Lung function normal between episodes }\end{array}$ & Intermittent & Mild persistent & Moderate persistent & Severe persistent \\
\hline $\begin{array}{l}\text { Step } 2 \\
\text { Symptoms }>1 \text { time per week } \\
\text { Nocturnal symptoms }<1 \text { time per week } \\
\text { Lung function normal between episodes }\end{array}$ & Mild persistent & Moderate persistent & Severe persistent & Severe persistent \\
\hline $\begin{array}{l}\text { Step } 3 \\
\text { Symptoms daily } \\
\text { Nocturnal symptoms } \geqslant 1 \text { time per week } \\
\text { FEV1 } 60-80 \% \text { pred }\end{array}$ & Moderate persistent & Severe persistent & Severe persistent & Severe persistent \\
\hline $\begin{array}{l}\text { Step } 4 \\
\text { Symptoms daily } \\
\text { Frequent nocturnal symptoms } \\
\text { FEV } 1 \leqslant 60 \% \text { pred }\end{array}$ & Severe persistent & Severe persistent & Severe persistent & Severe persistent \\
\hline
\end{tabular}

BDP: beclometasone dipropionate; LABA: Iong-acting $\beta_{2}$-agonist; FEV1: forced expiratory volume in one second; \% pred: \% predicted. Adapted from [4] with permission from the publisher.

Anti-IgE (omalizumab) was included in the 2004 update of the GINA guidelines (published 2005) and is the only add-on to step 4 therapy with category A evidence (rich body of evidence from randomised controlled trials). OCS may also be added to high-dose ICS/LABA therapy, but there are reservations about long-term use of such agents due to their association with significant side-effects, which include reversible abnormalities

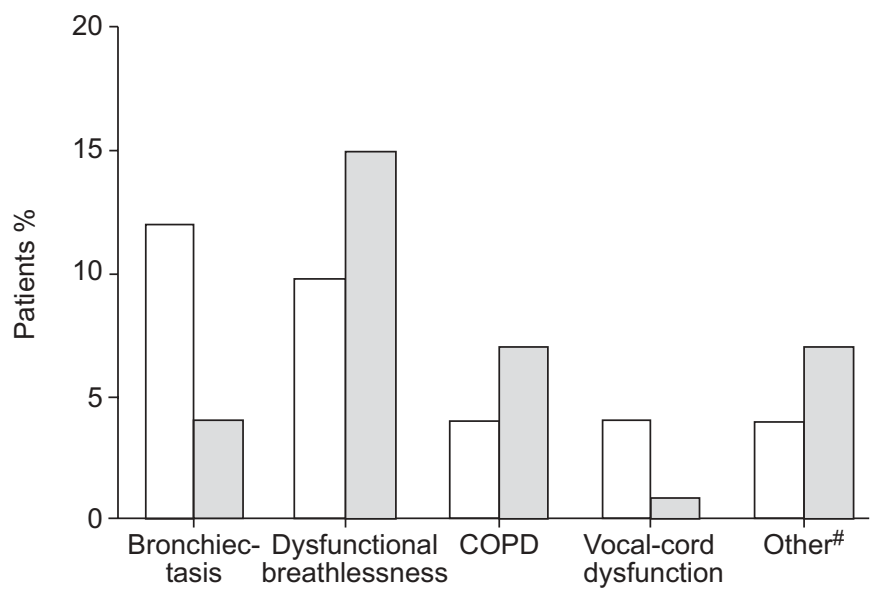

FIGURE 1. Proportion of patients with alternative or coexistent diagnosis causing symptoms in the Belfast $(\square)$ and Brompton ( $\square$ ) studies. COPD: chronic

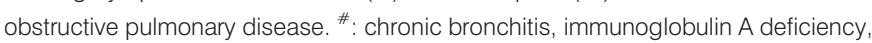
cystic fibrosis, obliterative bronchiolitis, cardiomyopathy, pulmonary hypertension, hypereosinophilia, extrinsic allergic alveolitis and respiratory muscle incoordination. Reproduced from [7] with permission from the publisher. in glucose metabolism, increased appetite, fluid retention, weight gain, rounding of the face, mood alteration, hypertension, peptic ulcer and aseptic necrosis of the femur. Theophylline and oral $\beta_{2}$-agonists are also possible additional add-on therapies to high-dose ICS in combination with LABA for use in patients with severe asthma, but are used much less frequently in this patient population due to comparatively limited evidence of efficacy and gastrointestinal and cardiovascular side-effects. Evidence for the efficacy of leukotriene modifiers in patients with severe asthma is limited.

GINA guidelines define asthma as controlled when there are minimal chronic symptoms, minimal (infrequent) exacerbations, no emergency visits, minimal use of as-needed SABA, no limitations on activities, a daily PEF variation of $<20 \%$, a nearnormal PEF and minimal adverse effects from medications. In severe persistent asthma, the goal of therapy is to achieve the best possible results: the fewest symptoms, the lowest requirement for inhaled $\mathrm{SABA}$, the best $\mathrm{PEF}$, the least circadian (night-to-day) variation and the fewest side-effects from medication.

\section{THE UNMET NEED IN SEVERE ASTHMA}

Suboptimal asthma management has been demonstrated in several studies, including the Asthma Insights and Reality in Europe (AIRE) study [14], the Fighting for Breath survey [15], the International Asthma Patient Insight Research (INSPIRE) study [16] and the Gaining Optimal Asthma Control (GOAL) study [17].

In the AIRE study [14], asthma patients were identified by telephone screening 73,880 households in seven European 


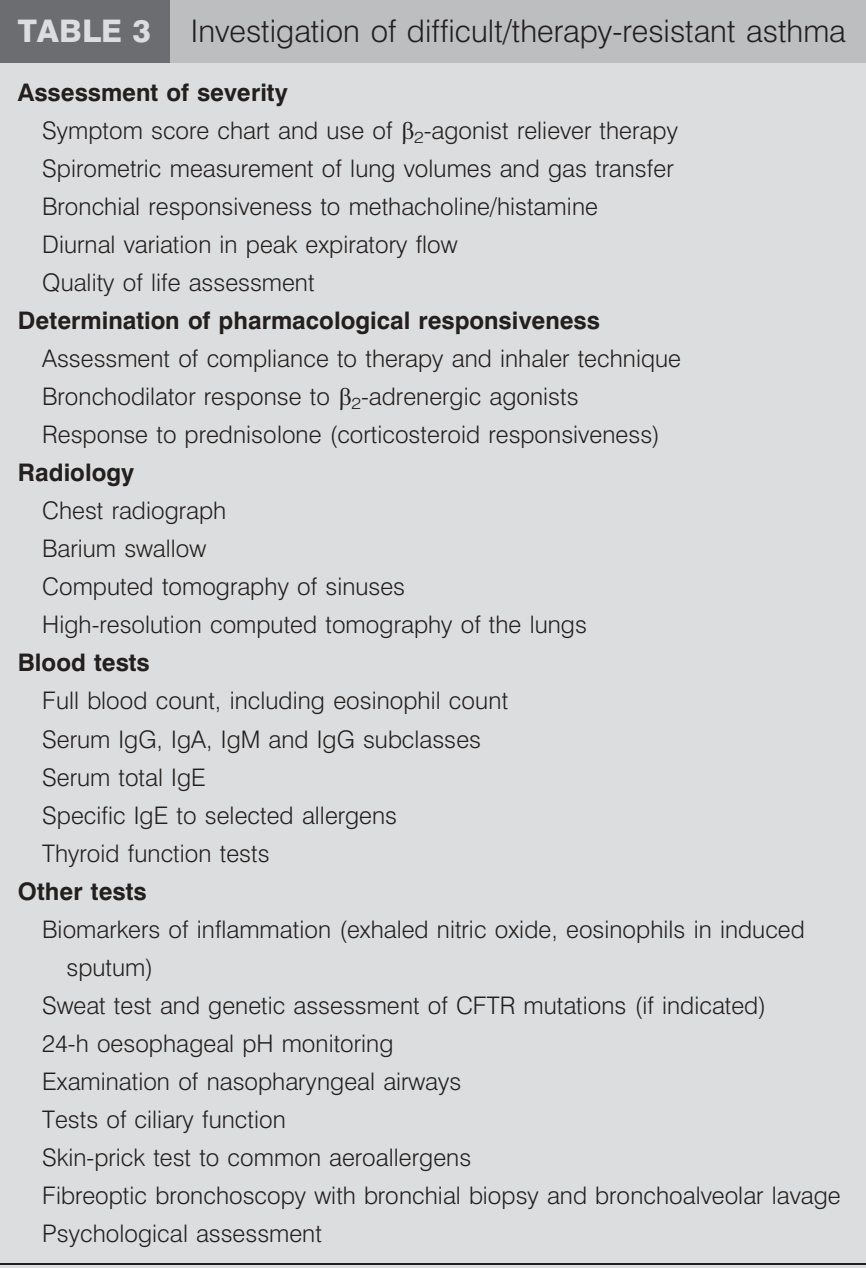

Ig: immunoglobulin; CFTR: cystic fibrosis transmembrane conductance regulator. Reproduced from [8] with permission from the publisher.

countries. Designated respondents were interviewed on healthcare utilisation, symptom severity, activity limitations and asthma control. Current asthma patients were identified in 3,488 households and 2,803 (80.4\%) patients completed the survey. The AIRE study showed that asthma is frequently poorly controlled and that levels of control do not meet the goals of the GINA guidelines. For example, $46 \%$ of patients reported daytime symptoms, 30\% had asthma-related sleep disturbances at least once a week, $25 \%$ of patients with asthma had an unscheduled urgent care visit in the previous year, $10 \%$ had an emergency room visit and $7 \%$ had an overnight hospitalisation. However, it is important to note that not all of these patients were following the correct level of medication for their severity of asthma. For example, only $\sim 30 \%$ of patients with moderate-to-severe persistent asthma were taking ICS [14].

The European Fighting for Breath survey of severe asthma [15] highlighted issues faced by asthma sufferers and estimated that 6 million Europeans with asthma live in fear that their next attack could kill them. Almost $70 \%$ have to restrict their physical activities, one in five felt disadvantaged at work or study and one in three felt restricted from going out socially.

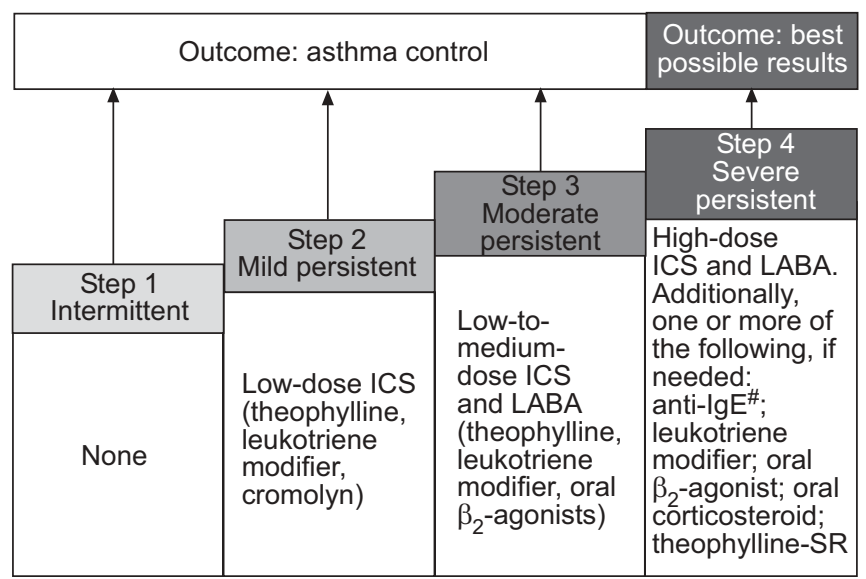

FIGURE 2. Global Initiative for Asthma 2005 guidelines stepwise approach to asthma control. Use of short-acting $\beta_{2}$-agonists to relieve symptoms is recommended at all treatment steps. ICS: inhaled corticosteroids; LABA: long-acting $\beta_{2}$ agonists; Ig: immunoglobulin; SR: sustained release. ${ }^{*}$ : anti-IgE is the only step 4 add-on therapy with category A evidence. Data taken from [4].

Others expressed how they felt ignored by society, encaged by their asthma and that they live with a sense of shame and embarrassment.

The INSPIRE study [16] examined the attitudes and actions of 3,415 physician-recruited adults aged $\geqslant 16$ yrs with asthma in 11 countries who were prescribed regular maintenance therapy with ICS alone or ICS and LABA. Structured interviews were conducted in order to assess medication use, asthma control and the patients' ability to recognise and self-manage worsening asthma. The respondents had a mean age of $45 \mathrm{yrs}$, their mean duration of asthma was $16 \mathrm{yrs}, 21 \%$ were smokers and $65 \%$ were female. All patients were receiving ICS and $70 \%$ were receiving ICS and a LABA $(61 \%$ in a fixed combination and $9 \%$ using separate inhalers). Despite receiving regular ICS or ICS/LABA maintenance therapy, $51 \%$ of asthma patients had uncontrolled symptoms as defined by the Asthma Control Questionnaire (ACQ; fig. 3), 74\% of patients used SABA rescue therapy daily and $51 \%$ of patients reported having needed unplanned medical care (such as hospitalisation) as a result of an asthma attack on at least one occasion in the previous year. Worsening asthma symptoms were found to affect all aspects of patients' daily lives, particularly leisure and social commitments. More than $70 \%$ of patients stated that the worst drawbacks of having asthma were the interference in their daily lives and the panic they felt as their symptoms increased, indicating the impact of the disease on the patients' healthrelated quality of life. Of the patients with asthma classified as "not well controlled" according to the ACQ, 87\% described their asthma control during the week preceding the interview as "relatively good", while $55 \%$ of patients classified as having "uncontrolled asthma" rated their asthma control as being "relatively good". This suggests that patients' notion of acceptable asthma control falls short of the level of control achievable. The INSPIRE study [16] also showed that although patients recognise deteriorating asthma control and adjust their medication during episodes of worsening, they often adjust treatment in a suboptimal manner, such as increasing SABA use, representing a missed window of opportunity. 


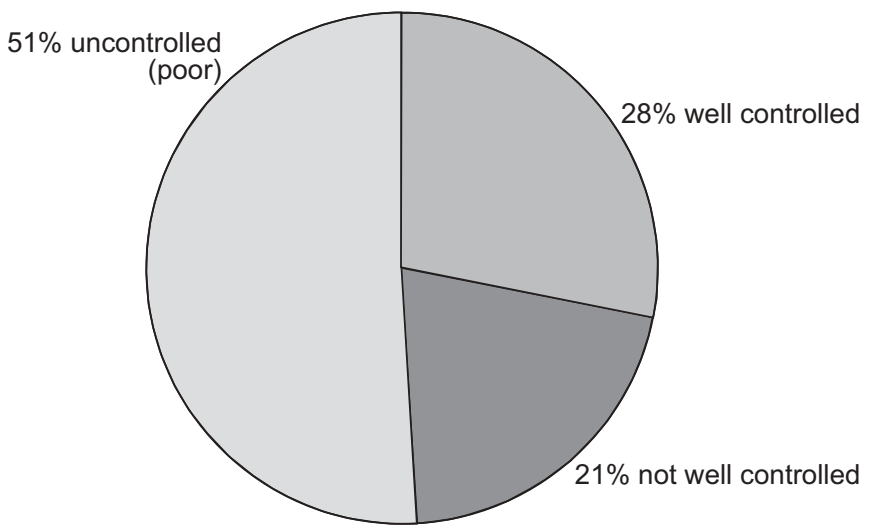

FIGURE 3. The International Asthma Patient Insight Research (INSPIRE) study. Only $28 \%$ of patients were well controlled according to the Asthma Control Questionnaire (ACQ). ACQ-6 summary score: well controlled: 0.0-0.74; not well controlled: 0.75-1.5; uncontrolled: >1.5. Data taken from [16].

The GOAL study [17] examined whether asthma control, as defined by guidelines, was achievable for most patients. This was a 1-yr, randomised, stratified, double-blind, parallel-group study of 3,421 patients with uncontrolled asthma and compared fluticasone propionate and salmeterol/fluticasone in achieving two rigorous, composite, guideline-based measures of control: total asthma control and well-controlled asthma. Treatment was stepped up until total control was achieved (or to a maximum of $500 \mu \mathrm{g}$ fluticasone b.i.d.). The GOAL study demonstrated that (in a controlled study) better guideline-derived asthma control is achievable in a majority of patients and that ICS/LABA is more effective than ICS alone. However, asthma was not well controlled in $38 \%$ of patients with the most severe asthma $\left(>500-1,000 \mu \mathrm{g} \cdot\right.$ day $^{-1}$ beclometasone equivalent at baseline) despite optimised ICS/LABA combination therapy (fig. 4). Furthermore, total asthma control was only achieved in $30 \%$ of patients with the most severe asthma (fig. 4). Adding OCS had little effect on asthma control, with asthma remaining inadequately controlled in $31 \%$ of patients with the most severe asthma and the proportion of patients with total control rising only slightly from $30 \%$ to $35 \%$.

\section{PATIENTS WITH SEVERE ASTHMA HAVE INCREASED RATES OF UNSCHEDULED HEALTHCARE}

A study that aimed to obtain a representative national picture of the type of people with asthma attending accident and emergency departments in the UK found that $425(32.9 \%)$ of the attendees had been admitted in the previous 12 months and $316(24.5 \%)$ had attended the emergency department in the previous 3 months [18]. Similarly, in a French multicentre trial of individuals with asthma attending emergency departments, $975(26 \%)$ out of 3,772 patients were considered to have lifethreatening asthma, $340(37 \%)$ had been hospitalised in the previous year and $75(8 \%)$ had been previously ventilated [19]. Patients with severe asthma account for the majority of hospitalisations due to asthma, and a strong association exists between asthma severity/hospitalisation and asthma-related mortality [20-25]. While all patients are susceptible to exacerbations, severe asthma increases the risk of an exacerbation being life-threatening or fatal $[26,27]$. In a 3-yr, multicentre,

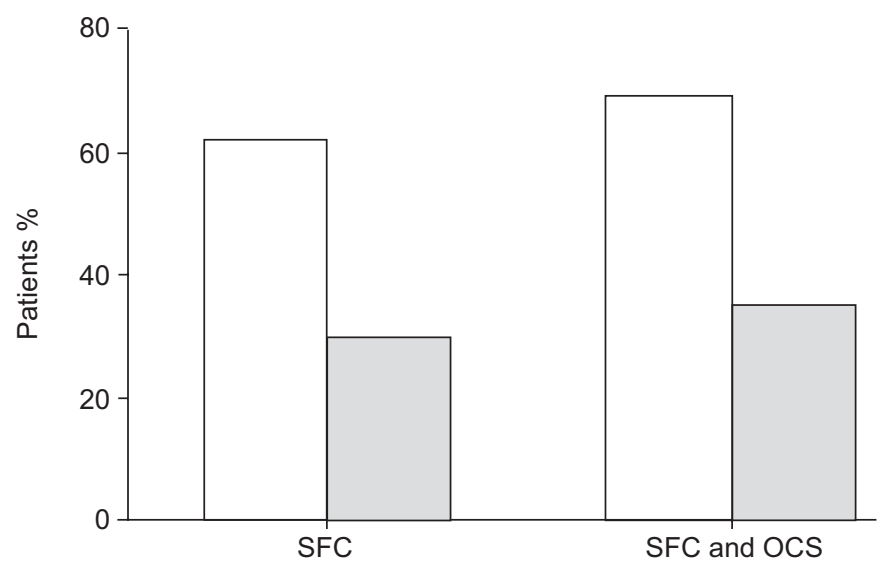

FIGURE 4. The Gaining Optimal Asthma Control (GOAL) study: $38 \%$ of

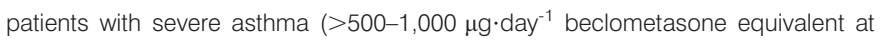
baseline) have symptoms that remain inadequately controlled despite optimised treatment with salmeterol/fluticasone combination therapy (SFC; $n=568$ ). $\square$ : well controlled at $1 \mathrm{yr}$; $:$ total control at $1 \mathrm{yr}$. OCS: oral corticosteroids. Data taken from [17].

observational study of almost 5,000 patients with severe or difficult-to-treat asthma in the USA (the Epidemiology and Natural History of Asthma: Outcomes and Treatment Regimens (TENOR) study), patients with severe asthma had considerably higher levels of healthcare utilisation than patients with mild or moderate asthma (fig. 5) [28].

\section{CONCLUSIONS}

Despite treatment with currently available therapy, there remains a patient group who have ongoing morbidity, require significant use of health services and are at risk of severe exacerbations. Omalizumab may help address the clear and unmet need for an effective and safe treatment of patients with severe persistent allergic asthma who remain symptomatic despite optimised standard treatment.

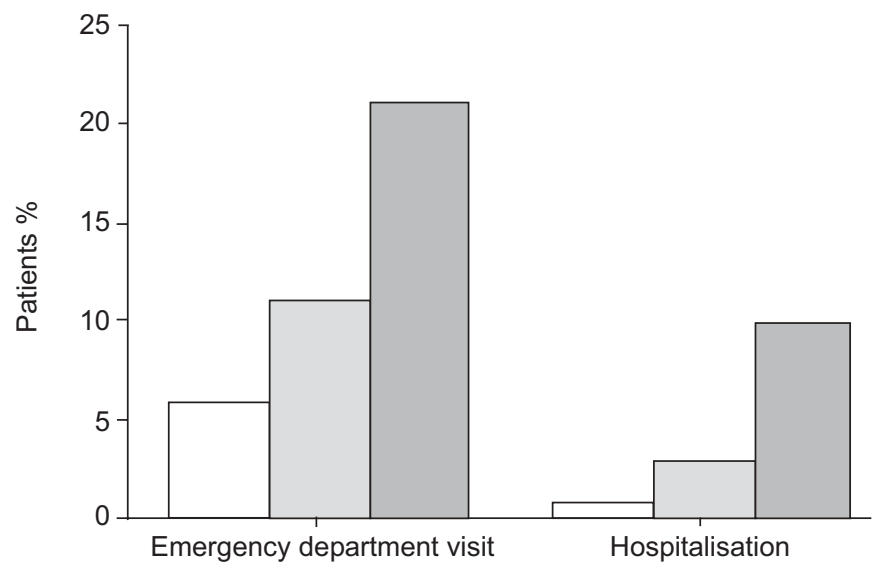

FIGURE 5. The group of patients with the most severe asthma has the highest incidence of hospitalisation and emergency department visits. $\square$ : mild asthma $(\mathrm{n}=219) ; \boldsymbol{a}$ : moderate $(\mathrm{n}=2,285) ; \mathbf{\square}$ : severe $(\mathrm{n}=2,285)$. Data taken from [28]. 


\section{REFERENCES}

1 Masoli M, Fabian D, Holt S, Beasley R, Global Initiative for Asthma (GINA) Program. The global burden of asthma: executive summary of the GINA Dissemination Committee report. Allergy 2004; 59: 469-478.

2 World Health Organization. The World Health Report 2003-Shaping the Future. Geneva, World Health Organization, 2003.

3 Loddenkemper R, Gibson GJ, Sibille Y, eds. European Respiratory Society/European Lung Foundation. Lung Health in Europe Facts and Figures. Sheffield, ERSJ, 2003.

4 Global Initiative for Asthma. Global strategy for asthma management and prevention. NIH Publication No 02-3659. Bethesda, National Institutes of Health/National Heart, Lung, and Blood Institute, 2005.

5 Robinson DS, Campbell DA, Durham SR, et al. Systematic assessment of difficult-to-treat asthma. Eur Respir J 2003; 22: 478-483.

6 Heaney LG, Conway E, Kelly C, et al. Predictors of therapy resistant asthma: outcome of a systematic evaluation protocol. Thorax 2003; 58: 561-566.

7 Heaney LG, Robinson DS. Severe asthma treatment: need for characterising patients. Lancet 2005; 365: 974-976.

8 Chung KF, Godard P, Adelroth E, et al. Difficult/therapyresistant asthma: the need for an integrated approach to define clinical phenotypes, evaluate risk factors, understand pathophysiology and find novel therapies. ERS Task Force on Difficult/Therapy-Resistant Asthma. European Respiratory Society. Eur Respir J 1999; 13: 1198-1208.

9 Irwin RS, Curley FJ, French CL. Difficult-to-control asthma. Contributing factors and outcome of a systematic management protocol. Chest 1993; 103: 1662-1669.

10 Morris MJ, Deal LE, Bean DR, Grbach VX, Morgan JA. Vocal cord dysfunction in patients with exertional dyspnea. Chest 1999; 116: 1676-1682.

11 Newman KB, Mason UG, Schmaling KB. Clinical features of vocal cord dysfunction. Am J Respir Crit Care Med 1995; 152: 1382-1386.

12 Mikita J, Parker J. High levels of medical utilization by ambulatory patients with vocal cord dysfunction as compared to age- and gender-matched asthmatics. Chest 2006; 129: 905-908.

13 Roberts NJ, Robinson DS, Partridge MR. How is difficult asthma managed? Eur Respir J 2006; 28: 968-973.

14 Rabe KF, Vermeire PA, Soriano JB, Maier WC. Clinical management of asthma in 1999: the Asthma Insights and Reality in Europe (AIRE) study. Eur Respir J 2000; 16: 802-807.

15 European Federation of Allergy and Airways Diseases Patients Associations. Fighting for Breath. www.efanet.org Date last accessed: October 2006.
16 Partridge MR, van der Molen T, Myrseth SE, Busse WW. Attitudes and actions of asthma patients on regular maintenance therapy: the INSPIRE study. BMC Pulm Med 2006; 6: 13.

17 Bateman ED, Boushey HA, Bousquet J, et al. Can guidelinedefined asthma control be achieved? The Gaining Optimal Asthma ControL study. Am J Respir Crit Care Med 2004; 170: 836-844.

18 Partridge MR, Latouche D, Trako E, Thurston JG. A national census of those attending UK accident and emergency departments with asthma. The UK National Asthma Task Force. J Accid Emerg Med 1997; 114: 16-20.

19 Salmeron S, Liard R, Elkharrat D, Muir J, Neukirch F, Elldrodt A. Asthma severity and adequacy of management in accident and emergency departments in France: a prospective study. Lancet 2001; 358: 629-635.

20 Taylor WR, Newacheck PW. Impact of childhood asthma on health. Pediatrics 1992; 90: 657-662.

21 Weber EJ, Silverman RA, Callaham ML, et al. A prospective multicenter study of factors associated with hospital admission among adults with acute asthma. Am J Med 2002; 113: 371-378.

22 Hartert TV, Speroff T, Togias A, et al. Risk factors for recurrent asthma hospital visits and death among a population of indigent older adults with asthma. Ann Allergy Asthma Immunol 2002; 89: 467-473.

23 Tough SC, Hessel PA, Ruff M, Green FH, Mitchell I, Butt JC. Features that distinguish those who die from asthma from community controls with asthma. J Asthma 1998; 35: 657-665.

24 Turner MO, Noertjojo K, Vedal S, Bai T, Crump S, Fitzgerald JM. Risk factors for near-fatal asthma. A casecontrol study in hospitalized patients with asthma. Am J Respir Crit Care Med 1998; 157: 1804-1809.

25 Rea HH, Scragg R, Jackson R, Beaglehole R, Fenwick J, Sutherland DC. A case-control study of deaths from asthma. Thorax 1986; 41: 833-839.

26 Burr ML, Davies BH, Hoare A, et al. A confidential inquiry into asthma deaths in Wales. Thorax 1999; 54: 985-989.

27 Jalaludin BB, Smith MA, Chey T, Orr NJ, Smith WT, Leeder SR. Risk factors for asthma deaths: a populationbased, case-control study. Aust N Z J Public Health 1999; 23: 595-600.

28 Dolan CM, Fraher KE, Bleecker ER, et al. Design and baseline characteristics of the epidemiology and natural history of asthma: Outcomes and Treatment Regimens (TENOR) study: a large cohort of patients with severe or difficult-to-treat asthma. Ann Allergy Asthma Immunol 2004; 92: 32-39. 